\title{
Approximate Solution for Longitudinal Vibration of Non-Uniform Beams by Differential Transform Method (DTM)
}

\author{
Keivan Torabi, Hassan Afshari and Ehsan Zafari \\ Department of Mechanical Engineering, University of Kashan, Kashan, Iran
}

Received: May 22, 2011 / Accepted: September 17, 2012 / Published: January 25, 2013.

\begin{abstract}
In this paper, differential transform method is applied for longitudinal vibration analysis of beams with non-uniform cross section. Natural frequencies and corresponding normalized mode shapes are calculated for different cases in cross section and boundary conditions. Comparison of results with the previous solutions proves the accuracy and versatility of the presented paper.
\end{abstract}

Key words: Differential transform method (DTM), longitudinal vibration, non-uniform beams.

\section{Introduction}

The differential transform method (DTM) is a numerical approach for solving linear, quasi-linear and some nonlinear, ordinary and partial differential equations. Many problems in engineering are described by differential equations; Most of these equations cannot be solved or are so difficult to solve analytically. Unfortunately, because of complexities which appear in solving differential equation of longitudinal vibration of beams, analytical methods can be used only for some special cases; Bapat [1] obtained the closed-form solution for the longitudinal vibration of beams whose cross section varies as a exponential function. Closed form solutions for longitudinal vibration of beams whose cross-section varies as $A(x)=A_{0}[1+a x / L]^{2}$ presented by Abrate [2]. Kumar and Sujith [3] derived exact solutions for the longitudinal vibration of non-uniform beams whose cross-section varies as $A(x)=(a+b x)^{n}$ and

Corresponding author: Keivan Torabi, assistant professor, research field: dynamic and control. E-mail: Kvntrb@kashanu.ac.ir.
$A(x)=A_{0} \sin ^{2}(a+b x)$. Li [4] combined the transfer matrix method and closed form solution of one step non-uniform rod to obtain a single frequency equation for a multi-step non-uniform rod. In another papers, he derived an exact solution for free longitudinal vibrations of one-step non-uniform rods with classical and non-classical boundary conditions [5] and an analytical solution for determine the longitudinal natural frequencies and mode shapes for a one step non-uniform bar with several boundary conditions [6]. $\mathrm{Li}$, et al. [7] found closed form solution for the longitudinal vibration of non-uniform structures with lumped masses and spring supports whose cross-section varies as $A(x)=\alpha(1+\beta x / L)^{n}$ and $A(x)=\alpha e^{-\beta x / L}$. Eigen frequencies for longitudinal vibration of inhomogeneous rods with certain area variations were obtained by Raj and Sujith for classical boundary conditions [8].

The concept of differential transform method was introduced first by Zhou [9], and it was applied to solve linear and nonlinear initial value problems in electrical circuit analysis. After that, DTM was applied to solve several problems in engineering. Using DTM, Chen 
and Ho $[10,11]$ solved eigenvalue problem for the free transverse vibration analysis of a rotating twisted Timoshenko beam under axial load. DTM was hired by Jang and Chen to solve a second order non-linear differential equation that describes the under damped and over damped motion of a system subject to external excitations [12]. In another paper, they used this method to solve the initial-value problems [13]. The DTM was used to find the dimensionless natural frequencies of the tapered cantilever Bernoulli-Euler beam by Ozdemir and Kaya [14]. Free vibration differential equations of motion of one end-fixed, the other simply supported and axial loaded beams on elastic soil was solved using DTM by Catal [15]. Arikoglu and Ozkol [16] used DTM to vibration analysis of composite sandwich beams with viscoelastic core. Ebaid [17] revealed that the DTM with the aftertreatment technique is very effective for a class of nonlinear oscillatory problems with fractional nonlinearities without any need for Padé approximants or Laplace transform.

In this paper, the application of the differential transform method is extended to acquire approximate solution for free longitudinal vibrations of non-uniform beams with arbitrary cross section. Comparison of results with the previous solutions proves the accuracy and versatility of the presented paper.

\section{Longitudinal Vibration of Beams}

The governing differential equation for the longitudinal vibration of a beam can be written as [18]:

$$
\frac{\partial}{\partial x}\left[E A(x) \frac{\partial w(x, t)}{\partial x}\right]-\rho A(x) \frac{\partial w(x, t)}{\partial t^{2}}=f(x, t)
$$

where $\rho$ and $E$ are mass per unit volume and Young's modulus of the beam, respectively; $A(\mathrm{x})$ is the area of section and $f(x, t)$ is the intensity of axial force. For free vibration analysis, $f(x, t)$ should be considered as zero and Eq. (1) can be simplified as:

$$
\frac{\partial}{\partial x}\left[E A(x) \frac{\partial w(x, t)}{\partial x}\right]-\rho A(x) \frac{\partial w(x, t)}{\partial t^{2}}=0
$$

The longitudinal displacement $w(x, t)$ can be assumed as the product of the function $u(x)$ which depends only on the spatial coordinate $x$ and $a$ time dependent harmonic function as $w(x, t)=u(x) \mathrm{e}^{i \omega t}$. Now, Eq. (2) can be expanded as:

$$
E A(x) \frac{d^{2} u(x)}{d x^{2}}+E \frac{d A(x)}{d x} \frac{d u(x)}{d x}+\rho A(x) \omega^{2} u(x)=0 .
$$

By considering dimensionless spatial coordinates as:

$$
\zeta=\frac{x}{L}
$$

Eq. (3) can be rewritten in the following dimensionless form:

$$
A(\zeta) \frac{d^{2} u(\zeta)}{d \zeta^{2}}+\frac{d A(\zeta)}{d \zeta} \frac{d u(\zeta)}{d \zeta}+\lambda^{2} A(\zeta) u(\zeta)=0
$$

where

$$
\lambda^{2}=\frac{\rho l^{2} \omega^{2}}{E}
$$

\section{Differential Transform Method (DTM)}

The differential transform method is a numerical method which uses the form of polynomials as an approximation of the exact solution. The differential transform method provides an iterative approach to obtain higher-order series. Basic definition and operation of DTM are introduced as below.

Differential transform of the function $u(\zeta)$ is defined around point $\zeta=\zeta_{0}$, as:

$$
U(k)=\left.\frac{1}{k !}\left[\frac{d^{k} u(\zeta)}{d \zeta^{k}}\right]\right|_{\zeta=\zeta_{0}}
$$

In Eq. (7), $u(\zeta)$ is the original function and $U(k)$ is transformed function. The function $u(\zeta)$ may be expressed in terms of the differential transform $U(k)$ as:

$$
u(\zeta)=\sum_{k=0}^{\infty}\left(\zeta-\zeta_{0}\right)^{k} U(k)
$$

Eq. (8) is known as the inverse differential transform of U(k). Substituting Eq. (7) into Eq. (8) gives:

$$
u(\zeta)=\left.\sum_{k=0}^{\infty} \frac{\left(\zeta-\zeta_{0}\right)^{k}}{k !}\left[\frac{d^{k} u(\zeta)}{d \zeta^{k}}\right]\right|_{x=x_{0}}
$$

Eq. (9) indicates that the concept of differential transform is derived from Taylor's series expansion.

Actually, the function $u(\zeta)$ is expressed by a finite series and Eq. (8) should be written as: 
Table 1 Mathematical operations performed by DTM.

\begin{tabular}{ll}
\hline Original function & Transformed function \\
\hline$u(x)=\alpha y(x)+\beta z(x)$ & $U(k)=\alpha Y(k)+\beta Z(k)$ \\
$u(x)=\frac{d y(x)}{d x}$ & $U(k)=(k+1) Y(k+1)$ \\
$u(x)=\frac{d^{2} y(x)}{d x^{2}}$ & $U(k)=(k+1)(k+2) Y(k+2)$ \\
$u(x)=y(x) z(x)$ & $U(k)=\sum_{l=0}^{k} Y(l) Z(k-l)$ \\
$u(x)=x^{m}$ & $U(k)=\delta(k-m)= \begin{cases}1 & k=m \\
0 & k \neq m\end{cases}$ \\
\hline
\end{tabular}

Table 2 Dimensionless frequencies for first three modes of a cantilever conical beam.

\begin{tabular}{llll}
\hline & $\mathrm{DTM}(\mathrm{N}=21)$ & Exact solution [7] & Error (\%) \\
\hline$\lambda_{1}$ & 2.5730 & 2.5704 & 0.0010 \\
$\lambda_{2}$ & 5.3648 & 5.3540 & 0.0020 \\
$\lambda_{3}$ & 8.3000 & 8.3029 & 0.0003 \\
\hline
\end{tabular}

$$
u(\zeta)=\sum_{k=0}^{N}\left(\zeta-\zeta_{0}\right)^{k} U(k)
$$

Eq. (10) denotes that:

$$
u(\zeta)=\sum_{k=N+1}^{\infty}\left(\zeta-\zeta_{0}\right)^{k} U(k)
$$

is negligibly small. In fact, the value of $N$ should be determined as:

$$
\left|\frac{\lambda_{i}^{(N)}-\lambda_{i}^{(N-1)}}{\lambda_{i}^{(N-1)}}\right| \leq \varepsilon
$$

Notice that for all numerical cases presented in this paper, convergence has been satisfied for $\varepsilon=0.01$.

Table 1 depicts the fundamental mathematical operations in $\zeta_{0}=0$ which performed by differential transform method.

\section{Numerical Results}

In this section some numerical examples are presented for various cases in cross section and boundary conditions.

\subsection{Cantilever Conical Beam}

Consider a cantilever tapered beam whose cross section varies $A(\zeta)=A_{0}(1-0.8 \zeta)^{2}$. For this example,
Eq. (5) can be written as:

$$
\left(0.64 \zeta^{2}-1.6 \zeta+1\right) \frac{d^{2} u}{d \zeta^{2}}+(1.28 \zeta-1.6) \frac{d u}{d \zeta}+\lambda^{2}\left(0.64 \zeta^{2}-1.6 \zeta+1\right) u=0
$$

and boundary conditions can be considered as:

$$
\begin{gathered}
u(0)=0 \\
\left.\frac{d u}{d \zeta}\right|_{\zeta=0}=0 .
\end{gathered}
$$

Transformed form of Eq. (12) can be written as:

$$
\begin{aligned}
& 0.64 \sum_{l=0}^{k} \delta(l-2)(k-l+1)(k-l+2) U(k-l+2) \\
& -1.6 \sum_{l=0}^{k} \delta(l-1)(k-l+1)(k-l+2) U(k-l+2) \\
& +(k+1)(k+2) U(k+2) \\
& +1.28 \sum_{l=0}^{k} \delta(l-1)(k-l+1) U(k-l+1)-1.6(k+1) U(k+1) \\
& +\lambda^{2}\left[0.64 \sum_{l=0}^{k} \delta(l-2) U(k-l)-1.6 \sum_{l=0}^{k} \delta(l-1) U(k-l)+U(k)\right]=0
\end{aligned}
$$

and in similar manner, boundary conditions can be written in the following transformed form:

$$
\begin{aligned}
& U(0)=0 \\
& \sum_{k=0}^{N} k U(k)=0
\end{aligned}
$$

Eq. (14) can be written as follows:

$$
\begin{aligned}
& U(k+2)= \\
& \left\{1.6(k+1)^{2} U(k+1)-\left[0.64 k(k+1)+\lambda^{2}\right] U(k)\right. \\
& \left.+\lambda^{2}[1.6 U(k-1)-0.64 U(k-2)]\right\} /(k+1)(k+2) .
\end{aligned}
$$

Using Eq. (16), further values of $U(k)$ can be derived as a function of $U(1)$. Substituting this values into Eq. (15b), characteristic equation can be obtained in term of dimensionless frequency.

Table 2 shows values of the first three dimensionless frequencies and corresponding values of the exact solution [7]. Substituting values of $\mathrm{U}(\mathrm{k})$ into Eq. (8), the corresponding modes will be obtained. These mode shapes are presented in Appendix A. It is worth mentioning that all mode shapes are normalized as follows:

$$
\bar{u}_{i}(\zeta)=u_{i}(\zeta) / \max \left|u_{i}(\zeta)\right|
$$

According to Ref. [7], exact modes can be derived for this example as: 


$$
\begin{gathered}
u_{i}(\zeta)=\left\{\cos \left[\lambda_{i}(1-0.8 \zeta)\right]\right. \\
\left.-\sin \left[\lambda_{i}(1-0.8 \zeta)\right] / \tan \left(\lambda_{i}\right)\right\} /(1-0.8 \zeta)
\end{gathered}
$$

For this case, the first three normalized mode shapes, are presented in Appendix A, These modes are depicted in Fig. 1.

\subsection{Clamped-clamped Conical Beam Whose Cross} Section Varies Exponentially

Consider a clamped-clamped beam whose cross section varies as $\mathrm{A}(\zeta)=A_{0} \mathrm{e}^{-\zeta}$. For this example, Eq. (5) can be written as:

$$
\frac{d^{2} u}{d \zeta^{2}}-\frac{d u}{d \zeta}+\lambda^{2} u=0
$$

and boundary conditions can be imposed as:

$$
\begin{aligned}
& u(0)=0 \\
& u(1)=0 .
\end{aligned}
$$

Eq. (19) can be written in DTM form as

$$
(k+1)(k+2) U(k+2)-(k+1) U(k+1)+\lambda^{2} U(k)=0
$$

and in similar manner, DTM form of boundary conditions can be written as follows:

$$
\begin{aligned}
& U(0)=0 \\
& \sum_{k=0}^{N} U(k)=0
\end{aligned}
$$

Eq. (21) can be written as:

$$
U(k+2)=\left[(k+1) U(k+1)-\lambda^{2} U(k)\right] /(k+1)(k+2)
$$

In similar manner, characteristic equation, dimensionless frequencies and corresponding normal modes can be derived. First lowest dimensionless frequencies are presented in Table 3. According to Ref. [7], exact modes can be derived for this example as:

$$
u_{i}(\zeta)=e^{0.5 \zeta} \sin (i \pi \zeta)
$$

For this case, the first three normalized mode shapes are presented in Appendix B for both DTM and exact solution; This modes are illustrated in Fig. 2.

\subsection{Cantilever Conical Beam with Sinusoidal Change in Diameter}

Now consider a cantilever beam which its diameter changes as $d(\zeta)=d_{0} \cos (\pi \zeta / 3)$ and therefore cross section of beam varies as $A(\zeta)=A_{0} \cos ^{2}(\pi \zeta / 3)$. In this case, Eq. (5) can be written as

$$
\cos ^{2}(\pi \zeta / 3) \frac{d^{2} u}{d \zeta^{2}}-\frac{\pi}{3} \sin (2 \pi \zeta / 3) \frac{d u}{d \zeta}+\lambda^{2} \cos ^{2}(\pi \zeta / 3) u=0
$$

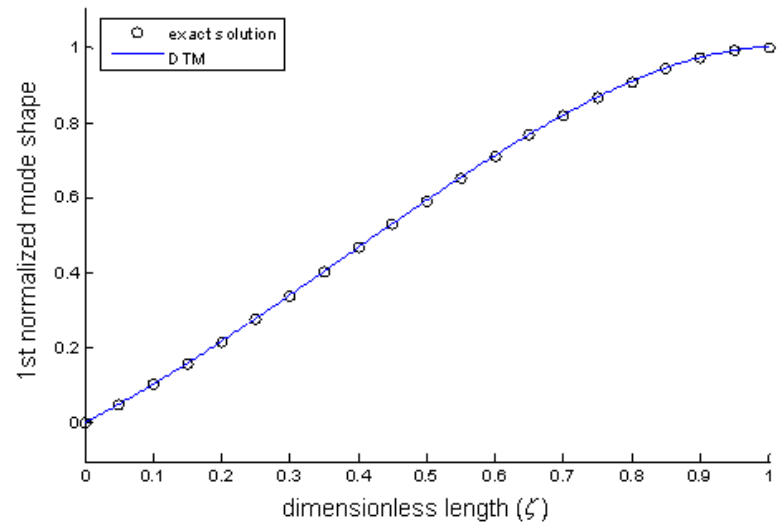

(a)

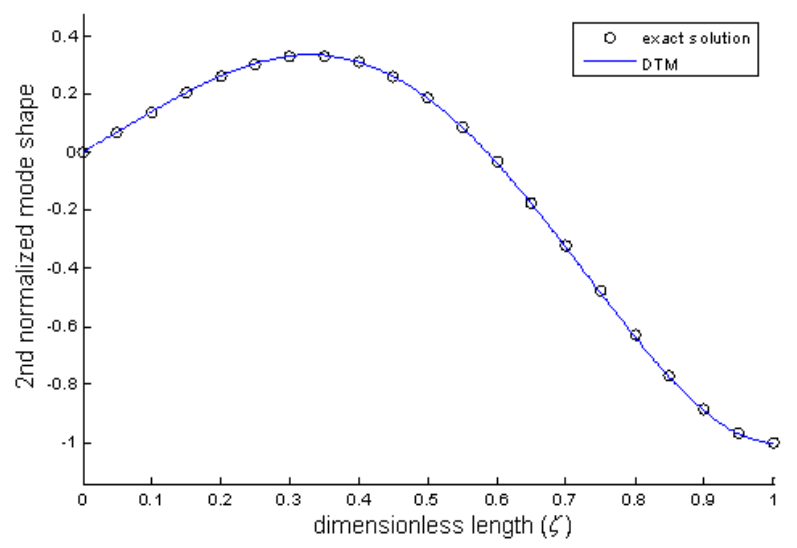

(b)

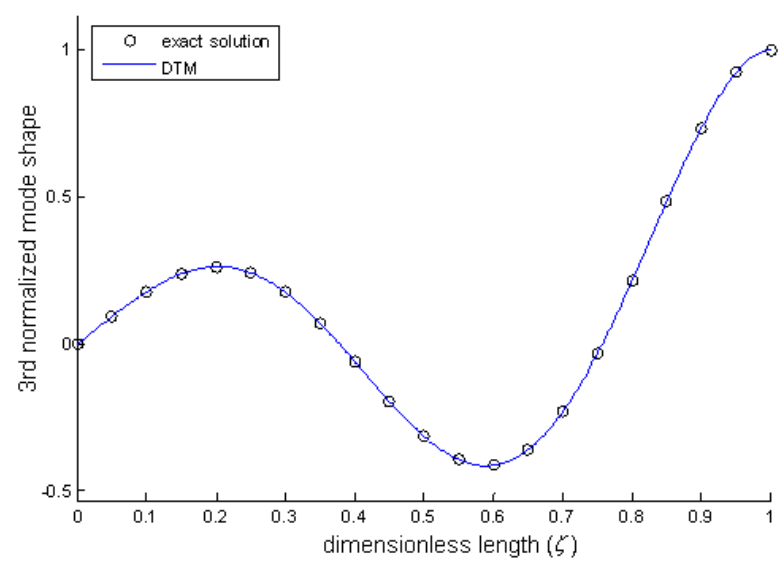

(c)

Fig. 1 (a) 1st normalized mode of cantilever conical beam, (b) 2nd normalized mode of cantilever conical beam, (c) 3rd normalized mode of cantilever conical beam. 


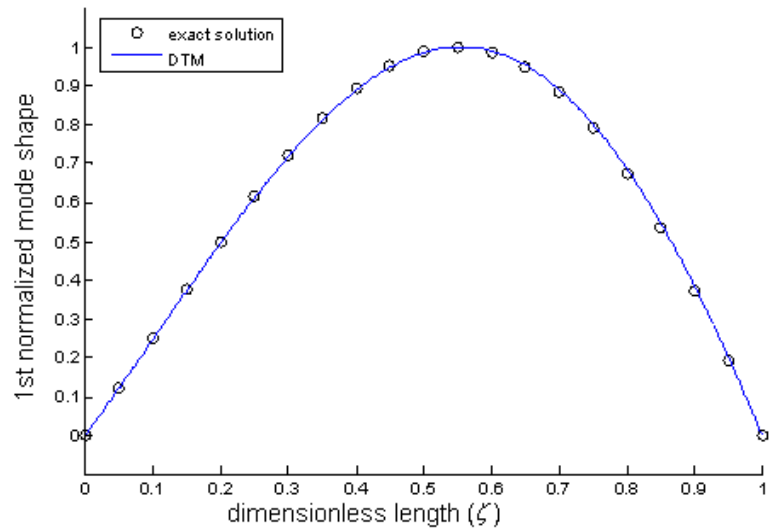

(a)

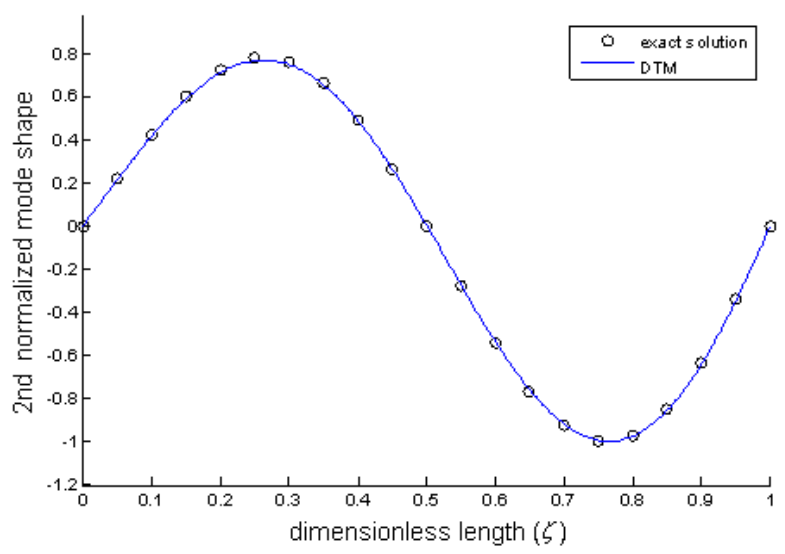

(b)

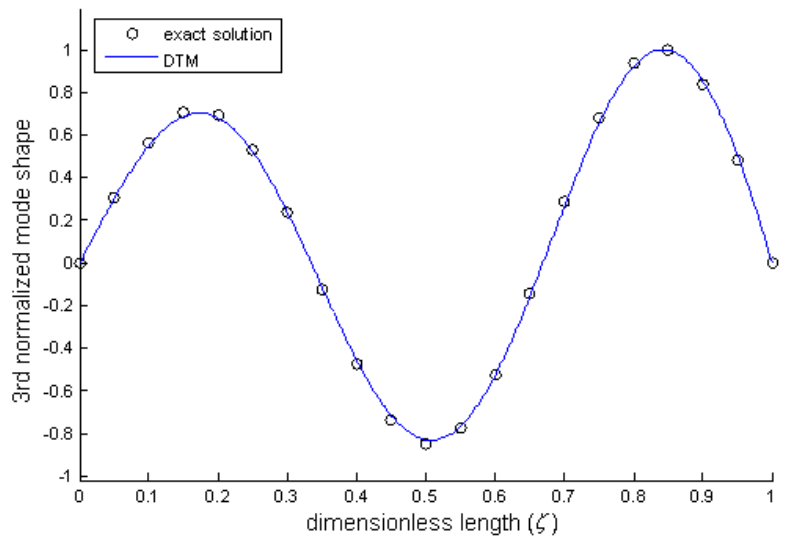

(c)

Fig. 2 (a) 1st normalized mode of clamped-clamped beam whose area changes exponentially. (b) 2nd normalized mode of clamped-clamped beam whose area changes exponentially. (c) 3rd normalized mode of clamped-clamped beam whose area changes exponentially.

To be able to utilize the mathematical operations which presented in Table 1, the Taylor's series expansion of sinusoidal functions should be used in Eq. (25). Now
Table 3 Dimensionless frequencies for first three modes of a clamped-clamped beam which area changes exponentially.

\begin{tabular}{llll}
\hline & $\mathrm{DTM}(\mathrm{N}=25)$ & Exact solution [7] & Error (\%) \\
\cline { 2 - 4 }$\lambda_{1}$ & 3.1811 & 3.1811 & 0 \\
$\lambda_{2}$ & 6.3030 & 6.3030 & 0 \\
$\lambda_{3}$ & 9.4122 & 9.4380 & 0.0027 \\
\hline
\end{tabular}

Table 4 Cantilever conical beam with sinusoidal change in diameter.

\begin{tabular}{llccc}
\hline $\mathrm{M}$ & 2 & 4 & 6 & 8 \\
\hline $\mathrm{N}$ & 16 & 12 & 12 & 12 \\
$\lambda_{1}$ & 2.2415 & 1.9568 & 1.9999 & 1.9733 \\
$\lambda_{2}$ & 5.5971 & 4.8597 & 5.0052 & 4.9891 \\
$\lambda_{3}$ & 6.8832 & 6.3052 & 6.4041 & 6.4189 \\
\hline
\end{tabular}

the degree of the polynomial which use for convergence $(\mathrm{N})$, depends on the degree of Taylor's series expansion (M). In the other words, convergence depends on both values of $\mathrm{M}$ and $\mathrm{N}$.

Table 4 shows the value of first three lowest dimensionless frequencies. Results show that in this case convergence obtains for $\mathrm{M}=8$ and $\mathrm{N}=12$. In other words if values of $\mathrm{N}$ and $\mathrm{M}$ increase, there is no sensible change in first three frequencies.

It is considerable that for high value of $\mathrm{M}$, say $\mathrm{M}=$ $20,30,40, \ldots$ convergence will be satisfied again for $\mathrm{N}$ $=12$; in other words after convergence, appropriate value of $\mathrm{N}$ will not change with increasing in value of M. After convergence the value of $\mathrm{N}$ is independent of $\mathrm{M}$, because by increasing the terms, there is no considerable change in error of the Taylor's series expansion.

\section{Conclusions}

Analytical methods are so limit to solve differential equations which appear in analysis of longitudinal vibration of non-uniform beams; therefore, the exact solutions have been presented for some special cases such as conical beam. Using DTM, corresponding differential equation can be solved for all cross-sections. The comparison between the results for the cases which have the exact solution shows that DTM is a strong method to solve differential equations. 
In cases which non-polynomial terms appear in differential equation, appropriate value of $\mathrm{N}$ that satisfied the convergence, depends on the value of $\mathrm{M}$ but after convergence, the value of $\mathrm{N}$ is independent of $\mathrm{M}$, because by increasing the terms, there is no considerable change in error of the Taylor's series expansion.

\section{References}

[1] C.N. Bapat, Vibration of rods with uniformly tapered sections, J. Sound Vib. 185 (1995) 185-189.

[2] S. Abrate, Vibration of non-uniform rods and beams, J. Sound Vib. 185 (1995) 703-716.

[3] B.M. Kumar, R.I. Sujith, Exact solutions for the longitudinal vibration of non-uniform rods, J. Sound Vib. 207 (5) (1997) 721-729.

[4] Q.S. Li, Exact solutions for free longitudinal vibration of stepped non-uniform rods, Appl. Acoust. 60 (2000) 13-28.

[5] Q.S. Li, Exact solutions for free longitudinal vibration of non-uniform rods, J. Sound Vib. 234 (1) (2000) 1-19.

[6] Q.S. Li, Free longitudinal vibration analysis of multi-step non-uniform bars based on piecewise analytical solutions, Eng. Struct. 22 (2000) 1205-1215.

[7] Q.S. Li, J.R. Wu, J. Xu, Longitudinal vibration of multi-step non-uniform structures with lumped masses and spring supports, Appl. Acoust. 333 (2002) 350-363.

[8] Anil Raj, R.I. Sujith, Closed-form solutions for the free longitudinal vibration of inhomogeneous rods, J. Sound Vib. 283 (2005) 1015-1030.

[9] J.K. Zhou, Differential Transform and Its Applications for
Electrical circuits (in chinese), Huazhong University press, Wuhan, China, 1986.

[10] C.K. Chen, S.H. Ho, Application of differential transformation to eigenvalue problem, J. Appl. Math. Comput. 79 (1996) 173-188.

[11] C.K. Chen, S.H. Ho, Transverse vibration of a rotating twisted Timoshenko beam under axial loading using differential transform, Int. J. Mech. Sci. 41 (1999) 1339-1356.

[12] M.J. Jang, C.L. Chen, Analysis of the response of a strongly non-linear damped system using a differential transformation technique, Appl. Math. Comput. 88 (1997) 137-151.

[13] M.J. Jang, C.L. Chen, Y.C. Liu, On solving the initial-value problems using differential transformation method, Appl. Math. Comput. 115 (2000) 145-160.

[14] O. Ozdemir, M.O. Kaya, Flabse bending vibration analysis of a rotating tapered cantilever Bernoulli-Euler beam by differential transform method, J. Sound Vib. 289 (2006) 413-420.

[15] S. Catal, Solution of free vibration equations of beam on elastic soil by using differential transform method, J. Appl. Math. Model. 32 (2008) 1744-1757.

[16] A. Arikoglu, I. Ozkol, Vibration analysis of composite sandwich beams with viscoelastic core by using differential transform method, J. Sound Vib. 92 (2010) 3031-3039.

[17] A.E. Ebaid, A reliable after treatment for improving the differential transformation method and its application to nonlinear oscillators with fractional nonlinearities, J. Nonlinear Sci. Numer. Simulat. 16 (2011) 528-536.

[18] C.W. De Silva, Vibration: Fundamentals and Practice, CRC Press, 2000, pp. 302-305.

\section{Appendix A}

Normalized modes of a cantilever conical beam.

DTM:

$$
\begin{aligned}
& \bar{u}_{1}(\zeta)=0.9547 \zeta+0.7638 \zeta^{2}-0.4424 \zeta^{3}-0.3539 \zeta^{4}+0.0656 \zeta^{5}+0.0524 \zeta^{6}-0.0130 \zeta^{7}-0.0104 \zeta^{8} \\
& -0.0033 \zeta^{9}-0.0026 \zeta^{10}-0.0024 \zeta^{11}-0.0019 \zeta^{12}-0.0015 \zeta^{13}-0.0012 \zeta^{14}-(9.7358 e-4) \zeta^{15} \\
& -(7.7886 e-4) \zeta^{16}-(6.2308 e-4) \zeta^{17}-(4.9846 e-4) \zeta^{18}-(3.9877 e-4) \zeta^{19}-(3.1902 e-4) \zeta^{20} \\
& -(2.5521 e-4) \zeta^{21} \\
& \bar{u}_{2}(\zeta)=-1.3452 \zeta-1.0762 \zeta^{2}+5.5918 \zeta^{3}+4.4735 \zeta^{4}-5.7072 \zeta^{5}-4.5658 \zeta^{6}+2.7108 \zeta^{7}+2.1686 \zeta^{8} \\
& -0.8088 \zeta^{9}-0.647 \zeta^{10}+0.1479 \zeta^{11}+0.1183 \zeta^{12}-0.0281 \zeta^{13}-0.0225 \zeta^{14}-0.0012 \zeta^{15}-(9.3423 e-4) \zeta^{16} \\
& -0.0025 \zeta^{17}-0.0020 \zeta^{18}-0.0015 \zeta^{19}-0.0012 \zeta^{20}-(9.499 e-4) \zeta^{21} \\
& \bar{u}_{3}(\zeta)=1.8452 \zeta+1.4761 \zeta^{2}-20.0046 \zeta^{3}-16.0037 \zeta^{4}+60.1701 \zeta^{5}+48.1361 \zeta^{6}-81.1835 \zeta^{7} \\
& -64.9468 \zeta^{8}+62.5641 \zeta^{9}+50.0513 \zeta^{10}-31.6802 \zeta^{11}-25.3442 \zeta^{12}+11.3967 \zeta^{13}+9.1174 \zeta^{14} \\
& -3.096 \zeta^{15}-2.4768 \zeta^{16}+0.65 \zeta^{17}+0.52 \zeta^{18}-0.114 \zeta^{19}-0.0912 \zeta^{20}+0.014 \zeta^{21}
\end{aligned}
$$


Exact solution:

$$
\begin{aligned}
& \bar{u}_{1}(\zeta)=\frac{0.1223}{1-0.8 \zeta}\{\cos [2.5704(1-0.8 \zeta)]+1.5561 \sin [2.5704(1-0.8 \zeta)]\} \\
& \bar{u}_{2}(\zeta)=\frac{-0.1762}{1-0.8 \zeta}\{\cos [5.3540(1-0.8 \zeta)]+0.7471 \sin [5.3540(1-0.8 \zeta)]\} \\
& \bar{u}_{3}(\zeta)=\frac{0.3746}{1-0.8 \zeta}\{\cos [8.3029(1-0.8 \zeta)]+0.4817 \sin [8.3029(1-0.8 \zeta)]\}
\end{aligned}
$$

\section{Appendix B}

Normalized modes of a clamped-clamped beam which area changes exponentially.

DTM:

$$
\begin{aligned}
& \bar{u}_{1}(\zeta)=2.416 \zeta+1.208 \zeta^{2}-3.6722 \zeta^{3}-1.9368 \zeta^{4}+1.4707 \zeta^{5}+0.8984 \zeta^{6}-0.226 \zeta^{7}-0.1906 \zeta^{8} \\
& +0.0106 \zeta^{9}+0.0225 \zeta^{10}+0.0011 \zeta^{11}+0.0016 \zeta^{12}-(1.9522 e-4) \zeta^{13}+(7.6975 e-5) \zeta^{14}+(1.4562 e-5) \zeta^{15} \\
& -(2.3293 e-6) \zeta^{16}-(6.8602 e-7) \zeta^{17}+(3.3734 e-8) \zeta^{18}+(2.175 e-8) \zeta^{19}+(9.8326 e-10) \zeta^{20} \\
& -(1.9319 e-10) \zeta^{21}-(4.0814 e-11) \zeta^{22}-(2.65 e-11) \zeta^{23}-(6.1009 e-12) \zeta^{24}+(8.5626 e-13) \zeta^{25} \\
& \bar{u}_{2}(\zeta)=-4.3053 \zeta-2.1527 \zeta^{2}+27.7897 \zeta^{3}+14.0743 \zeta^{4}-52.3873 \zeta^{5}-27.3695 \zeta^{6}+45.644 \zeta^{7} \\
& +25.1224 \zeta^{8}-22.3942 \zeta^{9}-13.3291 \zeta^{10}+6.8763 \zeta^{11}+4.5847 \zeta^{12}-1.3985 \zeta^{13}-1.1007 \zeta^{14} \\
& +0.1912 \zeta^{15}+0.1942 \zeta^{16}-0.0165 \zeta^{17}-0.0261 \zeta^{18}+(5.4205 e-004) \zeta^{19}+0.0028 \zeta^{20} \\
& +(8.0197 e-5) \zeta^{21}-(2.3348 e-4) \zeta^{22}-(1.647 e-5) \zeta^{23}+(1.6094 e-5) \zeta^{24}+(1.7345 e-6) \zeta^{25} \\
& \bar{u}_{3}(\zeta)=6.1827 \zeta+3.0913 \zeta^{2}-90.2567 \zeta^{3}-45.386 \zeta^{4}+390.7135 \zeta^{5}+199.1432 \zeta^{6}-795.6744 \zeta^{7} \\
& -414.4956 \zeta^{8}+932.9524 \zeta^{9}+501.2956 \zeta^{10}-705.7909 \zeta^{11}-395.2524 \zeta^{12}+370.4023 \zeta^{13} \\
& +218.849 \zeta^{14}-141.6664 \zeta^{15}-89.6365 \zeta^{16}+40.8676 \zeta^{17}+28.221 \zeta^{18}-9.1008 \zeta^{19} \\
& -7.0342 \zeta^{20}+1.5846 \zeta^{21}+1.4209 \zeta^{22}-0.2157 \zeta^{23}-0.237 \zeta^{24}+0.0224 \zeta^{25}
\end{aligned}
$$

Exact solution:

$$
\begin{gathered}
\bar{u}_{1}(\zeta)=0.769 e^{0.5 \zeta} \sin (\pi \zeta) \\
\bar{u}_{2}(\zeta)=-0.6852 e^{0.5 \zeta} \sin (2 \pi \zeta) \\
\bar{u}_{3}(\zeta)=0.6583 e^{0.5 \zeta} \sin (3 \pi \zeta)
\end{gathered}
$$

